\title{
COMUNIDAD INDUSTRIAL - VERSUCH EINER NEUEN STRUKTUR UND KONTROLLE DES EIGENTUMS IN DER PERUANISCHEN INDUSTRIE
}

\author{
Von Mechithild Minkner
}

Am 2. Oktober 1968 übernahm das Militär in Peru die Regierungsgewalt. Die neuen Machthaber bezeichneten sich von Anfang an als Revolutionsregierung der Streitkräfte. Sie erklärten als ihr Hauptziel, mittels grundlegender Reformen in den ökonomischen, sozialen, politischen und kulturellen Strukturen der peruanischen Gesellschaft die internen Machtverhältnisse zu verändern, die strukturellen Ungleichgewichte sowie die externe Abhängigkeit als Grundlagen zur Úberwindung der Unterentwicklung abzubauen. Die Regierung hat seit der Machtübernahme bei den verschiedensten Anlässen unterstrichen, daß das peruanische Modell einen Mittelweg zwischen Kapitalismus und Kommunismus suche, in dem eine Gesellschaft sich herausbildet, in der alle Gruppen und Schichten in voller Partizipation die Entwicklung bestimmen.

Na.ch den ersten tiefergehenden Reformmaßnahmen im Agrarselstor und im Bergbau erließ die peruanische Regierung am 27. 7. 1970 die Ley General de Industrias (Aligemeines Industriegesetz), der am 1. 9. 1970 die Ley de Comunidad Industrial1 folgte ${ }^{2}$. Beide Gesetze fixieren erste grundlegende Schritte zur strukturellen Neuordnung des industriellen Sektors. Die Ley General de Industrias (D. L. 18350 in Verbindung mit D. L. 19 262) enthält - neben Bestiminungen zur Durchsetzung der Industrialisierungsstrategie - in verschiedenen Artikeln verstreute Einzelelemente zur Eigentums- und Unternehmensreform in der Industrie. Als einer der Kernpunkte ist die Reformierung der Industrieunternehmung durch die Comunidad Industrial anzusehen. Die auf diese Weise geschaffene "propiedad privada reformada" (reformiertes Privateigentum) ist eine der vier Eigentums- und Herrschaftsformen in der pluralistisch angelegten Reformkonzeption Perus.

Im Gegensatz zur Industrialisierungsstrategie mittels Importsubstitution, massiven Einsatzes ausländischen Kapitals und ohne tiefgreifende strukturelle Reformmaßnahmen des „desarrollismo“--Modells der Regierung Belaúnde Terry stellt die Revolutionsregierung in D. L. 18350 als grundlegendes Prinzip fest, daß nur die permanente und sich selbst tragende Industrialisierung die Basis für eine kontinuierliche sozio-ökonomische Entwicklung und die Unabhängigkeit des Landes sein kann. Kernstück dieser Industrialisierungsstrategie ist die Entwicklung der Produktionszweige mit 1. Priorität (Grundstoffindustrie, Kapitalgüter), die durch eine möglichst vollständige Mobilisierung aller nationalen Ressourcen abgestützt werden soll.

\footnotetext{
* Die nachfolgenden Ausführungen basieren im wesentlichen auf den Gesetzestexten, auf Gesprächen mit Sekundärinformanten sowie internem Material verschiedener Institutionen, das nur zum Teil publiziert

1 Der Begriff Comunidad Industrial hat im Deutschen keine vollzutreffende inhaltliche Entsprechung.

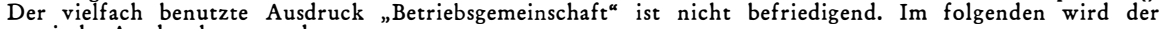
spanische Ausdruck verwandt.

2 Unter Industrie sind in diesem Zusammenhang alle Bereiche zu verstehen, die dem Ministerio de Industria y Turismo (MIT) zugeordnet sind. Die Fischmehlunternehmen, die Verarbeitungsbetriebe der 1. Stufe von Erdöl und Erdgas, Raffinerien und in sie integrierte Schmelzen von Nichteisenmetallen fallen unter die Verwaltung des Fischerei- bzw. Bergbauministeriums.
} 
Schwerpunkte der strukturellen Neuordnung sind:

- Gruppierung des Industriesektors in Unternehmen mit staatlichem, privatem ${ }^{3}$ und mit sozialem Eigentum ${ }^{4}$, denen spezielle Bereiche der industriellen Entwicklung als Aktionsfeld zugewiesen werden. Der Staat wird zum alleinigen Eigentümer aller Grundstoffindustrien (1. Priorität). Jedoch kann er mittels Konzessionsverträgen den anderen Eigentumsformen die Grundstoffindustrie als Betätigungsfeld einräumen. Er behält sich das Recht vor, in allen anderen Industriezweigen als alleiniger Kapitalgeber oder in assoziativer Form Unternehmen zu gründen.

- Einteilung der Industriezweige nach drei Prioritätsstufen und in eine nicht prioritäre Gruppe der Luxusgüter sowie selektive Förderung mittels fiskalischer, finanzieller und administrativer Maßnahmen.

- Fixierung der Operationsgrundlagen für Auslandskapital.

- Partizipation der Arbeitnehmer im Industriesektor mittels der Comunidad Industrial.

In der deutschsprachigen Literatur fehlen bis heute nicht nur detailliertere, kritische Analysen über die Strukturmaßnahmen im Industriesektor, sondern es mangelt auch an Abhandlungen mit mehr Informationscharakter. Grund dürfte nicht zuletzt die schmale Materialbasis über die Thematik sein. Von seiten peruanischer und nordamerikanischer Wissenschaftler wurden mehrere Arbeiten veröffentlicht, die auf der Grundlage mathematischer Modelle unter alternativen Annahmen zu bestimmen versuchen, in welchem Zeitraum die 50-Prozent-Grenze von der Comunidad Industrial erreicht wird. Über die ökonomischen, sozialen und politischen Auswirkungen der Comunidad Industrial liegen bis heute keine konsistenten empirisch belegten Ergebnisse vor. In den letzten Monaten wurden sowohl von offizieller Seite (Arbeitsgruppe für Spezialstudien des Ministerio de Industria y Turismo) als auch von privater Seite (Instituto de Estudios Peruanos) in Peru Arbeiten in Angriff genommen, die in die zuletzt genannte Richtung zielen.

Mit den nachfolgenden Ausführungen ist beabsichtigt, wein auch nur überblickweise, 1. den gesetzlichen Rahmen und die grundlegenders Bestimmungen der Comunidad Industrial darzustellen, 2. den Stand der Durchsetzung und die dabei auftretenden Konflikte unter den verschiedenen Gruppen im Unternehmen aufzuzeigen, 3. Tendenzen über geplante gesetzliche Veränderungen anzugeben, 4. Thesen über mögliche Auswirkungen und strukturelle Veränderungen zu entwickeln. Eine wissenschaftstheoretisch fundierte Analyse z. B. mittels des Hypothesenrahmens des Dependencia-Ansatzes ${ }^{5}$ oder eine Konfrontation der Comunidad Industrial mit den grundlegenden Thesen der „autogestión“ (Selbstbestimmung) können an dieser Stelle nicht geleistet werden.

3 Mit Einführung der Comunidad Industrial treten neben den privaten Bereich die „empresas de propiedad privada reformada" als vierter Subsektor. So auch vom Staatspräsidenten Velasco Alvarado in seiner Rede an die Nation am 28. 7. 1973 ausgeführt.

4 Das Gesetz über die "propiedad social“ (soziales Eigentum) wurde nach langer Diskussion und zeitlicher Verzögerung am 2. 5. 1974 erlassen (D. L. 20 598).

5 Der peruanische Soziologe A. Quijano versuchte eine erste Analyse des gesamten Modells, nicht nur der Reformmaßnahmen in der Industrie (vgl. Quijano, A., Nationalism and Capitalism in Peru: A Study in Neo-Imperialism; Monthly Review Press, New York, 1971). 


\section{Der gesetzliche Rahmen der Comunidad Industrial}

Gesetzliche Grundlage für die Comunidad Industrial sind die Ley General de Industrias (D. L. 18 350, geändert durch D. L. 19 262) und die Ley de Comunidad Industrial (D. L. 18 384). Als wichtigste Ergänzungsbestimmungen sind zu nennen:

- D. S. 023-71-EF, 17. 3. 1971

Präzisierung der Berechnung des zu versteuernden Gewinns.

- D. L. 19 339, 28. 3. 1972

Erklärt die Oficina de Comunidades Laborales (OCLA) dafür zuständig, die Comunidades Industriales zu koordinieren und zu beraten sowie die Organisation und Ausbildung durchzuführen.

- D. L. 19 340, 28. 3. 1972

Verfahren der Übernahme von Aktien durch die Comunidad Industrial und Finanzierungsmodus.

- D. S. 050-72-EF, 4. 4. 1972

Verlustabzüge und ähnliche abzugsfähige Posten zur Berechnung des steuerpflichtigen Gewinns.

- D. S. 021-72-CI/DS, 6. 6.1972

Der MIT wird in Koordinierung mit anderen Ministerien und/oder SINAMOS ${ }^{6}$ beauftragt, die Ausbildung der CI in die Hand zu nehmen.

- Resolution CNV-073-72-EF/94, 23. 8. 1972

Bewertungsgrundsätze und Schiedsinstanz bei der Transferierung von Aktien an die Comunidad Laboral6a.

- D. L. $20023,15.5 .1973$

Anpassung des Konkursgesetzes an die Bestimmungen über Comunidad Industrial: Möglichkeit der Übernahme der Unternehmensleitung eines in Konkurs befindlichen Unternehmens durch die Comunidad Industrial.

\section{Ziele, Funktionsweise und Organe der Comunidad Industrial}

D. L. 18384 (Art. 1 und 2) definiert die Comunidad Industrial als eine juristische Person des Privatrechts, die von allen dauerhaft und vollzeitlich beschäftigten Arbeitnehmern ${ }^{7}$ in einem Industriebetrieb gebildet wird, der 6 oder mehr Beschäftigte hat oder bei weniger als 6 Beschäftigten mehr als eine Million S/.8 Bruttoeinnahmen pro Jahr erzielt. Art. 12 fügt hinzu, daß die Arbeitnehmer tatsächlich (real y efectivamente) gegen Lohn oder Gehalt im Unternehmen arbeiten müssen ${ }^{8 a}$.

Als Ziele der Comunidad Industrial sind in Art. 3 festgelegt:

- Stärkung des Unternehmens;

6 Sistema Nacional de Apoyo a la Movilización Social.

6a Comunidad Laboral ist der Oberbegriff für die spezifischen Formen: Comunidad Industrial, Comunidad Pesquera, Comunidad Minera und Comunidad de Telecomunicaciones.

7 Das Gesetz präzisiert die Begriffe "dauerhaft" und "vollzeitlich“ nicht. In der Praxis der Comunidad Industrial wird als "dauerhaft ${ }^{*}$ definiert, wenn der A"rbeitnehmer nicht mehr in der Probezeit ist; nicht zum technischen Personal gehört, das spezielle, zeitlich begrenzte Verträge hat (Art. 55). Die zuletzt genannte Ausnahme widerspricht in gewisser Weise dem Grundprinzip, daß jeder Arbeitnehmer, ob genannte Ausnahme widerspricht in gewisser Weise dem Grundprinzip, daß jeder Arbeitnehmer, ob
mit unbegrenztem oder begrenztem Vertrag für die zeitliche Dauer Arbeitsstabilität genießt und damit, falls die anderen Bedingungen erfüllt werden, Mitglied der Comunidad Industrial ist. "Vollzeitlich“ kann als die vom Unternehmen festgesetzte Arbeitszeit ausgelegt werden. Der Ministerio de Industria y Turismo hat in Anlehnung an die Definition von "vollzeitlich“ bei Sozialleistungen entschieden, daß alle Arbeitnehmer, die mindestens vier Stunden beschäftigt sind, als vollzeitlich beschäftigt gelten.

8 Peruanische Währung Soles $=\mathrm{S} /$.

8a) Die Auslegung von "tatsächlich gearbeitet" ist ebenfalls umstritten. Es können einerseits die Tage sein, in denen der Arbeitnehmer seine Funktionen im Unternehmen tatsächlich erfüllt. Andererseits können die Tage, in denen er krank war, in denen er Urlaub hatte etc., hinzugerechnet werden. Das Ministerium regelte verschiedene Anfragen im zuletzt genannten Sinn. 
- Schaffung der Aktionseinheit der Arbeitnehmer in der Unternehmensführung, um ihre Interessen und Rechte als Miteigentümer zu schützen;

- Verwaltung des Vermögens der Arbeitnehmer;

- Förderung der sozialen, kulturellen und beruflichen Ausbildung der Arbeitnehmer.

Zur Stärkung der Unternehmen, die als langfristige Sicherung von Stabilität, Wachstum und Rentabilität interpretiert werden kann, soll die Comunidad Industrial einerseits mittels geeintem Vorgehen der Beschäftigten in bezug auf die Unternehmensführung, den Produktionsprozeß, das Eigentum am Unternehmen und die Reinvestition, andererseits mittels Förderung konstruktiver Beziehungen zwischen Arbeit und Kapital beitragen.

Abgesehen von der Spezifizierung der Zielsetzungen der Comunidad Industrial in Art. 3 geht aus Inhalt und Anlage des Gesetzes hervor, daß es darauf abzielt, das Unternehmen kapitalistischer Prägung zu verändern, indem dem Faktor Arbeit verschiedene Rechte zugesprochen werden, die sonst nur dem Faktor Kapital inhärent sind. Mittels der Comunidad Industrial soll die Partizipation des Faktors Arbeit am Eigentum, am Gewinn und an der Leitung des Unternehmens verwirklicht werden.

Die Beteiligung am Eigentum des Unternehmens geschieht mittels einer kontinuierlichen Vermögensbildung der Comunidad Industrial. Das Vermögen der Comunidad Industrial setzt sich gemäß Art. 16 in Verbindung mit Art. 19 wie folgt zusammen:

- Aktien oder Beteiligungen, die durch die sofortige und steuerfreie Kapitalisierung von steuerfreien Reinvestitionen (15 Prozent des steuerpflichtigen Nettogewinns des Unternehmens pro Jahr) gebildet und an die Comunidad Industrial als Vertretung des Faktors Arbeit übertragen werden bis 50 Prozent des Grundkapitals erreicht sind;

- Aktien oder Beteiligungen am bestehenden Grundkapital des Unternehmens, die mit dem 15prozentigen Anteil erworben werden, falls eine Reinvestition nicht in Frage kommt;

- Teile der Reinvestition des Unternehmens in anderen Unternehmen, die entsprechend dem Aktienanteil der Comunidad Industrial am eigenen Unternehmen auf sie entfallen und - allerdings nicht steuerfrei - kapitalisiert werden;

- Fondo General (Allgemeiner Fonds) ${ }^{9}$ der Comunidad Industrial, in dem Zinsen, Dividenden und - nach Erreichen der 50-Prozent-Grenze - die 15prozentige Beteiligung am steuerpflichtigen Nettogewinn akkumuliert werden ${ }^{10}$ sowie maximal 20 Prozent jährlich der Reserven, die für die „indemnización“ (Entschädigung der Arbeitnehmer bei Ausscheiden aus dem Unternehmen) vorgesehen sind, und zwar für den Fall, daß der Ordentliche Fonds zur „Auszahlung“ der ausscheidenden Arbeitnehmer aus der Comunidad Industrial nicht ausreicht ${ }^{11}$.

9 Die Mittel des Fondo General, der aus dem Fondo Ordinario und dem Fondo Excepcional gebildet wird, sind u. a. einzusetzen: für den Ankauf von Aktien und Rechten aus dem Betrieb ausscheidender Arbeitnehmer; zur Reinvestition in Aktien des eigenen Unternehmens, in Kapital neuer Unternehmen, in Staatspapiere; zur Zahlung von Dividenden, Zinsen etc. an die Mitglieder der Comunidad Industrial; zur Begleichung der administrativen Kosten sowie zur Förderung der allgemeinen und berufsspeziellen Ausbildung der "comuneros“.

10 Mittels dieser Äkkumulierung entsteht der Fondo Ordinario (Ordentlicher Fonds) als Teil des Fondo General (Art. 19).

11 Diese Mittel bilden den Fondo Excepcional (Außerordentlicher Fonds) der Comunidad Industrial (Art. 19). Es handelt sich bei diesen Mitteln praktisch um einen Kredit des Unternehmens an die Comunidad Industrial solange die Gelder des Fondo Ordinario noch nicht ausreichen. 
- Sonstige Vermögenstitel, die die Comunidad Industrial erwirbt.

Den beiden Grundformen der Vermögensbildung und -partizipation der Arbeitnehmer liegt als klar erkennbare Strategie zugrunde, die Unternehmen anzureizen, mittels Reinvestition die Kapitalbasis in der Industrie zu verbreitern und damit die Industrialisierung zu beschleunigen, auch wenn die 50-Prozent-Grenze damit langsamer erreicht wird. Entschließt sich das Unternehmen nicht zur Reinvestition bzw. wird diese nicht genehmigt, ermöglicht der Ankauf von Aktien und Anteilen der Kapitaleigner die 50-Prozent-Beteiligung in kürzerer Frist zu verwirklichen. Eine unkontrollierte, mit der Industrialisierungsstrategie und Industrieplanung nicht in Einklang stehende Reinvestition wird durch Art. 24 der Ley General de Industrias unterbunden, nach dem die Reinvestitionsprogramme dem Industrieministerium vorgelegt und von diesem gebiligt werden müssen. Auf Reinvestitionen in andere Unternehmen kann nur bedingt ausgewichen werden, da die Comunidad Industrial proportional ihrem Aktienanteil beteiligt wird.

Die Partizipation des Faktors Arbeit am Eigentum der Unternehmung zeichnet sich durch einige spezielle Regelungen aus (Art. 17, 18, 22):

- Die Vermögens- bzw. Eigentumsbeteiligung am Unternehmen erfolgt nicht individuell durch jeden Arbeitnehmer, sondern mittels der Comunidad Industrial, die alle Arbeitnehmer vertritt. Grundgedanke ist, die erwünschten Zielsetzungen der Comunidad Industrial nicht durch Zersplitterung des Faktors Arbeit zu verwässern.

- Die Vermögensanteile der Comunidad Industrial können nicht transferiert werden und als Garantie nur bei Operationen mit staatlichen Banken dienen. Damit ist die Möglichkeit ausgeschaltet, daß die Comunidad Industrial die Kapitalbeteiligung am Unternehmen verliert.

- Die Comunidad Industrial gibt erst nach Erreichen der 50-Prozent-Grenze über ihren Eigentumsanteil individuelle Aktien ${ }^{12}$ an die Arbeitnehmer aus, und zwar jeweils entsprechend der Anzahl der tatsächlich gearbeiteten Monate. Erst von diesem Zeitpunkt an empfängt der Arbeiter direkt die ihm dadurch zustehenden Anteile aus dem zur Verteilung stehenden Gewinn, falls sich das Unternehmen nicht zur Reinvestition oder Reservenbildung entschließt. Vor Erreichen der 50-Prozent-Grenze werden an die Beschäftigten 50 Prozent der ihnen dem Kapitalanteil entsprechend zustehenden Gewinne in gleichen Teilen ausgegeben und 50 Prozent gemäß den Arbeitsjahren seit Bestehen der Comunidad Industrial. Durch diese Differenzierung wird die Einheit des Faktors Arbeit, entscheidend für das Erreichen der 50-Prozent-Grenze, gestärkt.

Neben dem Recht der Gewinnbeteiligung, das sich aus dem Kapitaleigentum der Comunidad Industrial am Unternehmen ableitet, haben alle Arbeitnehmer, die tatsächlich und vollzeitlich im Unternehmen arbeiten, Anrecht auf 10 Prozent am steuerpflichtigen Gewinn (D. L. 18 350, Art. 21), der zu 50 Prozent zu gleichen Anteilen verteilt wird, zu 50 Prozent proportional der Basisentlohnung.

Als weiteres, erstmals den Arbeitnehmern der Industrie Perus zugestandenes Recht leitet sich aus der Kapitalbeteiligung des Faktors Arbeit die Partizipation an der Unternehmensführung $\mathrm{ab}^{\mathbf{1 3}}$. Mit dem Zeitpunkt der Anerkennung (Art. 243, D. S.

12 Der Wert jeder Aktie berechnet sich aus dem Gesamtwert der Aktien oder Beteiligungen der Comunidad Industrial dividiert durch die tatsächlich gearbeitete Zeit der Arbeitnehmer (Art. 22).

13 Mit D. L. 18366 wurde für alle Wirtschaftsbereiche außer Industrie, Bergbau, Fischerei und Massenmedien ein Gewinnbeteiligungssystem eingeführt, das neben der Comunidad Laboral fortbesteht. 
Nr. 007-71- IC/DS in Verbindung mit Art. 45, D. L. 18 384) entsendet die Comunidad Industrial einen Vertreter in den „directorio“ der AG ${ }^{14}$ bzw. das entsprechende Organ anderer juristischer Gesellschaftsformen. Die Zahl der Vertreter der Comunidad Industrial nimmt im „directorio“ der AG und unter den „socios colectivos" der KG auf Aktien proportional zur Erhöhung der Kapitalbeteiligung der Comunidad Industrial zu. Sobald die Comunidad 50 Prozent Kapitaleigentum erreicht und damit 50 Prozent der Mitglieder des „directorio“ stellt, muß eine Neuwahl des Vorsitzenden stattfinden (Art. 48, D. L. 18 384). In der GmbH ist die Comunidad nur durch einen Vertreter präsent, jedoch werden die Entscheidungen im Einklang mit den Anteilen der Kapitaleigner am Gesellschaftskapital vorgenommen. Vertreter der Comunidad Industrial in der Hauptversammlung ist vom Zeitpunkt des erstmaligen Erwerbs von Aktien gemäß Art. 45 der Präsident des Consejo der Comunidad Industrial. In diesem Unternehmensorgan nimmt die Zahl der Vertreter nicht zu, jedoch erhöht sich die Zahl der Stimmen mit zunehmendem Aktienanteil. Existiert ein Aufsichtsrat ${ }^{15}$ im Unternehmen, nimmt der Präsident der Comunidad Industrial auch dort die Vertretung wahr.

Bis zum Erreichen der 50-Prozent-Grenze haben die Vertreter der Comunidad Industrial eine konfliktreichere Position inne als die "normalen“ Minderheitsaktionäre. Sie vertreten die Interessen und üben die Rechte des Faktors Arbeit aus von dem sie gewählt und entsendet wurden - , die vielfach erheblich von denen der "socios capitalistas" divergieren bzw. von ihnen als der Kapitalmajorität verletzt werden dürften. Ein Minoritätenschutz, der gesetzlich nicht vorgesehen ist, könnte die Beteiligung an den grundlegenden Entscheidungen wie z. B. Auflösung, Kapitalerhöhung und Fusion effektiver gestalten.

Das Gesetz sieht keine Vertretung der Comunidad Industrial in der „gerencia"16, also unter den leitenden Angestellten vor. Von seiten der "comuneros" sieht man darin vielfach einen Widerspruch. Laut Art. 3 D. L. 18384 soll die Comunidad Industrial Einfluß auf den Produktionsprozeß und die Unternehmensentscheidungen nehmen. Die „täglichen" Entscheidungen der "gerencia“ können aber die Entwicklung der Unternehmung unter Umständen tiefgreifender beeinflussen als die des "directorio“, in dem die Comunidad präsent ist. In diesem Zusammenhang ist auch zu berücksichtigen, daß die "gerencia“ vom „directorio“ bestellt wird, falls die Statuten dieses Recht nicht der Aktionärsversammlung zusprechen. Es wäre widersinnig, der Comunidad Industrial in ihrer Eigenschaft als Kapitaleigner und Mitglied des „directorio“ weitergehende Rechte einzuräumen. Die Forderung der Comunidad Industrial, einen Vertreter in die "gerencia“ zu entsenden, geht u. a. von zwei Überlegungen aus. Die Comunidad hat, solange sie Minderheitsaktionär

14 Die Comunidad wählt und entsendet also ihren Vertreter im Gegensatz zu den anderen Mitgliedern des „directorio“, die von der Aktionärsversammlung gewählt werden. Der „directorio“ der peruanischen Aktiengesellschaft hat die rechtliche Vertretung der Gesellschaft und die "Geschäftsführung wahrzunehmen (Art. 162, Ley 16123). Er ist also mit dem Vorstand der AG vergleichbar, der nach deutschem Recht allerdings vom Aufsichtsrat bestellt wird.

15 Das Aufsichtsorgan der AG ist nach peruanischem Recht der „consejo de vigilancia“, z. T. vergleichbar mit dem Aufsichtsrat. Er wird nach deutschem und peruanischem Aktienrecht von der Aktionärsversammlung gewählt. Art. 188 der Ley 16123 bestimmt, daß alle Aktiengesellschaften mit mehr als 50 Aktionären sowie 20 Millionen S/. Grundkapital und mehr ein Aufsichtsorgan haben müssen. Der "consejo de vigilancia“ ist allerdings auch in den genannten Unternehmensgrößen nicht notwendig, wenn der "directorio" einstimmig gewählt wurde, mit Beteiligung der Minderheitsaktionäre besteht (Art. 158, Ley 16123) oder die Unternehmung permanent durch staatlich anerkannte Buchprüfer (contadores públicos colegiados) überwacht wird.

16 Art. 176 bis 187 der Ley 16123 legt Aufgaben und Funktionsweise der ngerencia“ fest. Aufgabe der "gerencia“ (sie kann aus einer oder mehreren Personen bestehen) ist die Durchführung aller ordentlichen Geschäfte und Verträge, die der Erfüllung des Unternehmensziels dienen (Art. 179). Die "gerencia“ ist der Gesellschaft, den Aktionären und Dritten für Schäden, die sich aus der Nichterfüllung etc. ihrer Aufgaben ergeben, Rechenschaft schuldig. 
ist, geringen Einfluß auf die Auswahl der "gerencia“. Der Funktionsbereich der "gerencia“, der für die einzelne Unternehmung in ihren Statuten präzisiert wird, kann sehr weit sein, vor allem dann, wenn sie das volle Vertrauen des "directorio“ genießt und dieser ihr weitgehend die eigenen Funktionen delegiert. Die Idee der direkten Einflußnahme in die "gerencia“ durch Beiordnung eines Vertreters der Comunidad Industrial wird damit plausibler. Im Zusammenhang mit diesen Fragen ist ferner zu bedenken, daß in großen Firmen das Management („gerencia“) sich als technokratische Schicht immer mehr verselbständigt und versucht, seine Tätigkeit weniger an den Interessenlagen der Eigentümer als an den reinen Unternehmenserfordernissen auszurichten. Als Beispiel sei die Gewinnverwendungspolitik angeführt. Die Einflußnahme der Comunidad Industrial, die sowohl Interessen des Faktors Arbeit als auch des Faktors Kapital vertritt, auf die "gerencia" ist auch aus diesem Grunde nicht sinnvoll. Zusätzliche Schwierigkeiten in der Steuerung des Unternehmensverlaufes können sich durch die Mitgliedschaft der „gerencia“ in der Comunidad Industrial ergeben, vor allem dann, wenn die leitenden Angestellten sehr eng mit den Kapitaleignern verbunden sind.

Die Leitung, Verwaltung und Kontrolle der Comunidad Industrial liegen in den Händen der Asamblea General und des Consejo der Comunidad Industrial (Art. 25 bis 37) ${ }^{17}$. Die Asamblea General, die zweimal im Jahr abgehalten werden muß18, wählt den Consejo, einschließlich des Präsidenten, billigt und kontrolliert die Arbeitsweise des Consejo, bestimmt über Gewinnverwendung sowie alle grundlegenden Angelegenheiten der Comunidad Industrial. Dem Consejo als Exekutivorgan obliegen die Verwaltung des Vermögens und die damit im Zusammenhang stehenden Aktivitäten der Comunidad Industrial. Die Zahl seiner Mitglieder, gewählt auf zwei Jahre mit Möglichkeit der Wiederwahl, wird in den Statuten festgelegt. Der Consejo benennt und entsendet einen oder mehrere Vertreter in den "directorio“ der AG bzw. das entsprechende Organ bei anderen Gesellschaftsformen.

Die Comunidad Industrial kommt in der analysierten Form nicht in allen Unternehmen der Industrie zur Anwendung (Art. 26 und 46). Spezielle Vorschriften bestehen bei den Industrien 1. Priorität für die Grundstoffindustrie und allen den Unternehmen, die als „strategisch“ für die nationale Entwicklung erklärt werden ${ }^{19}$. In diesen beiden Unternehmenstypen erwirbt die Comunidad Industrial mittels der $15 \%$ des steuerpflichtigen Jahresgewinns vom Unternehmen ausgegebene Bonds oder Aktien der Klasse $\mathrm{C}$ von COFIDE20. Diese Ausnahmeregelung hat entsprechende Auswirkung auf die Beteiligung am verteilungsfähigen, nicht am steuerpflichtigen Gewinn und an der Unternehmensführung. Die Comunidad Industrial erhält bei den Obligationen einen bestimmten Zinssatz pro Jahr. Er variiert also nicht mit der Höhe des zur Verteilung stehenden Gewinns des Unternehmens. Die Dividendenhöhe der Aktien Klasse C hängt allein von der Unternehmensund Gewinnlage von COFIDE ab, steht also in keinem Zusammenhang mit dem eigenen Unternehmensverlauf und -ergebnis. Das kann Vor- und Nachteile haben,

17 Die Asamblea General ist die Versammlung aller Mitglieder der Comunidad Industrial. Den Consejo könnte man als Verwaltungsgrat bezeichnen.

18 Sie kann als Außerordentliche Mitgliederversammlung auf Votum von ein Zehntel der Mitglieder oder des Consejo einberufen werden.

19 Art. 26 bestimmt, daß alle Unternehmen der 1. Priorität, die aufgrund des Produktes, der Investitionshöhe und des Technologieeinsatzes mittels Decreto Supremo als strategisch für die nationale Entwicklung erklärt werden, und in denen der Staat sich mit mindestens $30 \%$ und entscheidendem Einfluß in der Unternehmensführung mit nationalem oder ausländischem Kapital assoziiert, diese Ausnahmeregelung erlangen können.

20 Corporación Financiera de Desarrollo. 
entsprechend der positiven oder negativen Unternehmensentwicklung. Die Beteiligung an der Unternehmensführung ist aufgrund der unterschiedlichen Modalitäten der Vermögensbeteiligung ebenfalls beschränkt. Von Anfang an werden bei diesen Unternehmen zwei Vertreter in den Vorstand integriert. Eine Möglichkeit der Steigerung der Einflußnahme und Entscheidungsgewalt besteht nicht, da keine Beteiligung am Grundkapital vorgesehen ist. In der Aktionärsversammlung ist die Comunidad Industrial nicht präsent und hat damit keinen Einfluß auf die grundlegenden Unternehmensentscheidungen.

Grundidee dieser Ausnahmeregelung, die zu einer Diskriminierung und Differenzierung der Comunidades Industriales im Industriesektor führt, dürfte sein, daß der Staat „freie Hand“ in den für ihn strategischen Unternehmen behalten will. Ferner ist zu vermuten, daß man sich die Möglichkeit freihalten will, gerade dem Auslandskapital - das vielfach aufgrund der Comunidad Industrial sowie der sonstigen Beschneidung der Unternehmerfreiheit einer Investition skeptisch gegenübersteht - , sofern man an ihm stark interessiert ist, diese Sonderlösung anzubieten. Denn der Begriff „strategisch“ ist so unbestimmt, daß die verschiedensten Unternehmen darunter fallen können. Bayer Industrial S. A. (BISA), Tochtergesellschaft der Holding Bayer Foreign Investment Ltd., wurde mittels Decreto Supremo im Juni 1972 als gemischtes Unternehmen zur strategischen Industrie erklärt und unterliegt damit in der Partizipation des Faktors Arbeit den erwähnten Ausnahmen.

Die Sonderregelungen für „strategische Industrien“ wurden mit D. L. 19262 vom 6. 1. 1972 eingeführt. Die Sociedad Nacional de Industrias kommentiert dazu sibyllinisch, ob das System der Comunidad Industrial nicht vielleicht doch einige Irrtümer enthalte und den Interessenlagen und Entwicklungserfordernissen zuwiderlaufe, so daß Korrekturen für den staatlichen und gemischten Sektor angebracht scheinen, die der privaten Industrie aber nicht zugestanden werden ${ }^{21}$.

\section{Widersprüche und Lücken in Konzeption und Bestimmungen der Communidad Industrial}

Abgesehen von den Widerständen und Schwierigkeiten, die die Einführung und Durchsetzung dieser neuen Partizipationsform bei den Faktoren Kapital und Arbeit hervorruft, enthalten schon Konzeption und Gesetz eine Anzahl von Widersprüchen und Lücken, aus denen Konflikte verschiedenster Art entstehen müssen. Im folgenden sollen einige grundlegende Punkte aufgezeigt werden, ohne sie in ihren möglichen faktischen Konsequenzen zu diskutieren ${ }^{22}$.

Das Gesetz über die Comunidad Industrial billigt ausdrücklich dem Faktor Arbeit die Partizipation an Eigentum, Gewinn und Unternehmensführung zu. Die Umsetzung und Ausfüllung dieser Mitbestimmung regelt sich nach der Nueva Ley de Sociedades Mercantiles (Ley Nr. 16123 vom 6. 5. 1966), deren gesetzliche Normen auf das typisch kapitalistische Unternehmen, d. h. das alleinige Recht des Faktors Kapital über die Unternehmen zu bestimmen, zugeschnitten sind. Sie steht damit im Widerspruch zur Konzeption und den bestehenden Regelungen über die Comunidad Industrial.

21 Sociedad Nacional de Industrias, Industria Peruana, Januar 1972, S. 14

22 Sie werden z. T. an anderen Stellen der Ausführungen wieder aufgegriffen, z. T. wurden sie in der Darstellung und Analyse der grundlegenden gesetzlichen Bestimmungen angedeutet. 
- In der Philosophie der Revolutionsregierung wird eine volle und gleichmäßige Partizipation aller Bevölkerungskreise und damit auch aller Arbeitnehmer propagiert. Die Comunidad Industrial in ihrer jetzigen Form diskriminiert den Faktor Arbeit in kleinen, mittleren und großen Betrieben sowie in staatlichen, gemischten und privaten Unternehmen ${ }^{23}$. Die gesetzlichen Regelungen der Comunidad Laboral favorisieren die Arbeitnehmer in der Industrie ${ }^{24}$. Sie marginalisieren die Beschäftigten im Handels- und Dienstleistungssektor und zum Teil in der Landwirtschaft, wo eine derartige Partizipationsform nicht vorhanden ist ${ }^{25}$.

- An der Comunidad Industrial partizipieren nicht alle Beschäftigten eines Unternehmens, auch wenn sie eventuell entscheidend zum ökonomischen Ergebnis beitragen, wie das z. B. beim technischen Personal, das unter Zeitvertrag genommen wird, der Fall sein kann. Ausgleichsmechanismen sind nicht vorgesehen.

- Mitglied der Comunidad Industrial ist auch das Management der Unternehmung. Es wird vom Vorstand bestellt, ist also an die Weisungen, die sich aus der vom Vorstand konzipierten Unternehmenspolitik ergeben, letztlich gebunden. Obwohl Mitglied der Comunidad Industrial - die zumindest bis zur angestrebten Harmonisierung von Kapital und Arbeit vielfach vom Faktor Kapital divergierende Ziele anstrebt, als Beispiel sei hier nur die Lohnpolitik angeführt dürften seine Interessen sich also mehr der Unternehmerseite zuneigen. Konflikte mit den "comuneros" sind - auch aufgrund der privilegierten Stellung kaum zu vermeiden.

- Der Comunidad Industrial macht einerseits den Faktor Arbeit zum Miteigentümer, ausgestattet mit Rechten und Pflichten eines Kapitaleigentümers. Sie sieht den Posten eines Gwerkschaftsführers als nicht vereinbar mit dem eines Mitglieds des Consejo der Comunidad Industrial an. Gleichzeitig aber unterstreicht man, daß die Gewerkschaften ihre volle Berechtigung in der „empresa privada reformada" behalten. Divergenzen aus dieser Doppelrolle der Arbeitnehmer als Faktor und Kapital sind unvermeidbar.

Das Gesetz sieht eine 50:50-Beteiligung von "propietarios capitalistas26" und „propietarios comuneros ${ }^{27}$ im Stadium voller Verwirklichung des Modells vor. Das deutet an, wenn man eine rationale und kontinuierliche Unternehmensführung erhalten will, daß die Konflikte und Widersprüche zwischen Arbeit und Kapital aufgelöst sein müssen. Diese Annahme muß nach den bisherigen Erfahrungen, auch mit anderen Modellen, auf berechtigte $Z_{\text {weifel stoßen. }}$

- Das Gesetz sieht keine Durchführungsbestimmungen vor, um die bestehenden Lücken und Unstimmigkeiten auszufüllen und zu präzisieren. Das kann darauf hindeuten, daß das gesamte Modell nur als Übergangslösung konzipiert und der Beginn einer noch grundlegenderen Reform der Eigentums- und Unter-

23 Es bestehen nicht nur die erwähnten Sonderregelungen, sondern es wurde auch kein Ausgleichsfonds wie z. B. im Fischerei- und Bergbausektor geschaffen, um die Produktivitäts- und Rentabilitätsunterschiede zu kompensieren. 24 Die Mitglieder der Comunidad Minera partizipieren z. B. mit $4 \%$ bzw. $6 \%$ am steuerpflichtigen mit $10 \%$ bzw. $15 \%$. Die Rechte der Beteiligung an Kapital und Unternehmensführung sind ebenfalls abgestuft.

25 Die differenzierten Formen sind ökonomisch und politisch vom Standpunkt der Revolutionsregierung und den Entwicklungserfordernissen des Landes her möglicherweise sinnvoll. Sie schaffen jedoch neue privilegierte Gruppen.

26 Ursprüngliche Kapitaleigner.

27 Neue Kapitaleigner aufgrund der Comunidad Industrial. 
nehmensform im Industriesektor ist. Die Begründung der Propiedad Social (Sozialeigentum) mit D.L. 20598 vom 2. 5. 1974 bestätigt in gewisser Weise diese Annahme. Da die Propiedad Social von den Arbeitnehmern selbst bestimmt und höchste Förderungspriorität genießen wird, sind neue Konflikte zwischen den Gruppen zu erwarten ${ }^{28}$.

\section{Probleme der Durchsetzung der Comunidad Industrial}

\section{Entwicklung der Comunidad Industrial nach Zahl, Mitgliedern und Industriebranchen}

D. L. 18384 vom 1. 9. 1970 bestimmt in Art. 8, daß alle Unternehmen, die unter das Gesetz fallen, innerhalb von 60 Tagen die Comunidad Industrial begründen müssen. Die Gründungsversammlung ist vom höchsten administrativen Angestellten, also in der Regel dem Manager, einzuberufen und zu leiten. Das Gesetz legt die Initiative in die Hände der Unternehmensführung. Erst wenn diese nicht tätig wird, nimmt der MIT auf Antrag schon eines Arbeiters die Gründung vor.

Wie der Prozeß der Gründung der Comunidades Industriales de facto abgelaufen ist, darüber geben die folgenden Zahlen Aufschluß. Die Angaben aus Reden, Presseverlautbarungen, Archivmaterial und einigen Arbeitsdokumenten weichen geringfügig voneinander $a b^{29}$.

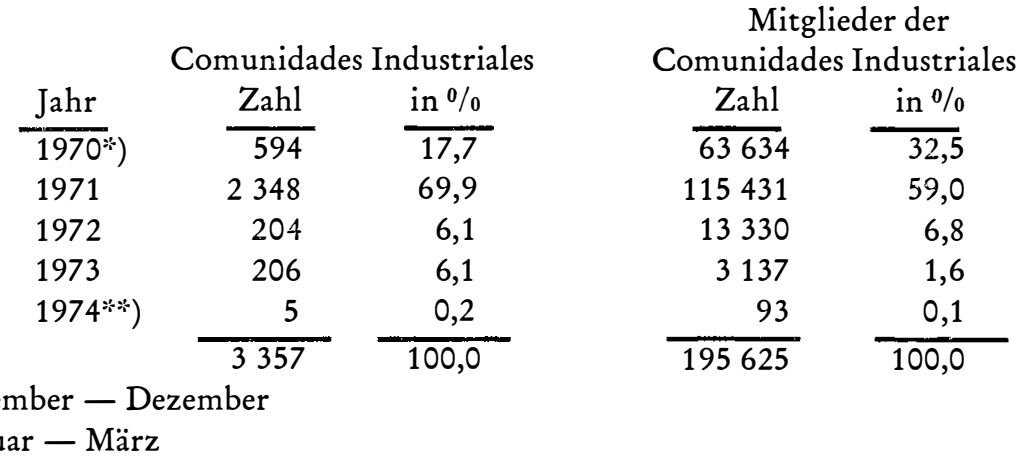

Nur etwa 1/5 aller bis zum 31. 3. 1974 anerkannten Comunidades Industriales fielen in das Jahr 1970; 70\% nahmen Gründung und Registrierung 1971 vor. Am Ende des zweiten Jahres nach Inkrafttreten des Gesetzes, also 1972, hatten mit 3146 Comunidades Industriales erst $63 \%$ aller der Unternehmen, die dafür in Frage kommen, die gesetzlichen Bestimmungen erfüllt. Der Prozentsatz steigerte sich bis Jahresende 1973 auf 90 . Noch mehr als drei Jahre nach Verkündung des D. L. 18384 ist ein "harter Kern“ von etwa 350 Unternehmen vorhanden, der keine Comunidad Industrial hat. Die Entwicklung der Mitgliederzahlen deutet an, daß die größeren Unternehmen, in denen der Druck der Arbeitnehmer und die Kontrolle durch die Öffentlichkeit vergleichsweise stärker sind als in Mittel- und

28 Nach Erlaß des D. L. 20598 häuften sich Pressemeldungen über Comunidades Industriales, die die Umwandlung der Unternehmung in Propiedad Social forderten.

29 Vgl. z. B. MIT, Principales discursos pronunciados en 1973 por el Contralmirante A. P. Alberto Jiménez de Lucio, Ministro de Industria y Turismo, Lima, Enero 1974, S. 84/85. 
Kleinbetrieben, in der Mehrzahl die Comunidad Industrial in den ersten drei Monaten eingeführt haben.

In der Verteilung der Comunidad Industrial nach Industriezweigen ${ }^{30}$ liegt die Nahrungsmittelbranche mit 16,4\% der Comunidades an der Spitze. Das entspricht dem Gewicht dieser traditionellen Industrie in der Struktur des Sekundärsektors. Nach der Anzahl der Comunidades folgen diverse Industrien (12\%), Konfektion und Schuhe $(11,5 \%)$, Textilien $(8,9 \%)$, Druckereien und Verlage $(8,0 \%)$, chemische Produkte $(7,3 \%)$, Metallprodukte ohne Transportmaterial $(6,6 \%)$, nichtmetallische Mineralien $(5,0 \%)$. Die anderen Industriezweige vereinigen Comunidades Industriales auf sich, die jeweils weniger als 5,0\% der Gesamtzahl ausmachen. Legt man die Zahl der „comuneros" zugrunde, steht die Textilindustrie (16,3\%) an erster Stelle. Es schließen sich Nahrungsmittel (13,2\%), chemische Produkte $(9,8 \%)$, Konfektion und Schuhe $(8,3 \%)$, diverse Industrien $(7,4 \%)$, nichtmineralische Metalle (5,7\%), Getränkeindustrie (5,3\%), Druckereien und Verlage (5,2\%) an. Die unterschiedliche Verteilung nach Industriebranchen der Zahl der Comunidades Industriales und der „comuneros“ hängt u. a. mit der differenzierten Betriebsgrößenstruktur zusammen. Die Einführung der Comunidad Industrial bereitet normalerweise in großen Betrieben mit relativ viel Beschäftigten weniger Schwierigkeiten als in mittleren und kleineren Unternehmen. Eine Branche, die nach der Unternehmenszahl nicht an der Spitze liegt, kann aufgrund der Betriebsgröße mehr comuneros aufweisen. Ein solcher Zusammenhang könnte z. B. bei den Branchen Nahrungsmittel, Textilien sowie chemische Produkte gegeben sein ${ }^{31}$. Die geographische Verteilung der anerkannten Comunidades Industriales entspricht der Standortkonzentration in der Industrie. Auf das Ballungsgebiet LimaCallao entfallen $81,7 \%$ der Comunidades Industriales mit $86 \%$ aller "comuneros“, gefolgt von Arequipa mit $4 \%$ bzw. 3,5\%.

\section{Die Beteiligung der Comunidad Industrial an Kapital und Gewinn der Unternehmen}

Die Anerkennung der Comunidad Industrial durch die Oficina de Comunidades Laborales (OCLA) des MIT sagt nichts über die faktische Durchsetzung der grundlegenden Rechte aus. Statistisch repräsentative Untersuchungen liegen darüber bis heute nicht vor. Die zur Verfügung stehenden Zahlen geben jedoch einen Anhaltspunkt über die Größenordnung der Probleme.

Aus einer Umfrage unter 332 Vertretern von Comunidades Industriales aus dem Jahre 1972 geht hervor, daß nur 55\% die Frage nach der Beteiligung am Aktienkapital positiv beantworten. Mit wachsender Größe nehmen die Widerstände ab, die Comunidad am Eigentum zu beteiligen. In den Unternehmen mit 6 bis 10 Beschäftigten erhielt die Comunidad Industrial nur in $38 \%$ der Fälle die ihr zustehenden Aktien; in den Unternehmen mit mehr als 200 Beschäftigten in $77 \%$ der Fälle. Die Frage nach der Gewinnbeteiligung wurde in rund $81 \%$ Fällen bejaht; die positive Quote erhöht sich wiederum mit wachsender Betriebsgröße. Von Ver-

\footnotetext{
30 Die Angaben beziehen sich auf Ende März 1974.
31 Nach Angaben des MIT betrug die durchschnittliche Beschäftigtenzahl 1970 in der Nahrungsmittelindustrie 25.3, in der Textilindustrie 46.7, in der chemischen Industrie 40.1 Personen (vgl. MIT, Estadística Industrial 1972, Lima, Diciembre 1973, S. 33, 57, 101).
} 
tretern aus Unternehmen mit 6 bis 10 Beschäftigten beantworteten nur $35 \%$ die Frage mit ja, von Unternehmen mit mehr als 200 Beschäftigten $87 \%$. Sowohl bei Durchsetzung der Kapital- als auch der Gewinnbeteiligung führen die gewerkschaftlich gut organisierten Branchen wie graphisches Gewerbe, Maschinen und Haushaltsgeräte.

Über die quantitative Entwicklung der Vermögens- und Gewinnbeteiligung in kapitalintensiven großen Betrieben geben einige Daten Aufschluß, die von 132 Unternehmen, die nur etwa $3 \%$ der Unternehmen insgesamt, deren Anlagevermögen und Bruttoproduktion jeweils aber etwa $50 \%$ des Sektors ausmachen, erhoben wurden ${ }^{32}$. Von ihnen waren $53 \%$ nationale, $16 \%$ gemischte und $31 \%$ ausländische Unternehmen.

Der Kapitalisierungsprozeß in den 132 Industriebetrieben wurde 1971/1972 durch drei Maßnahmen besonders beeinflußt:

- D. L. 18815 ermöglichte den Betrieben eine Neubewertung der Aktiva.

- D. L. 18350 führte ein neues System der Reservenkapitalisierung ein.

- D. L. 18350 billigte den Unternehmen eine spezielle Förderung für

Reinvestitionen zu.

Aufgrund der mit diesen Maßnahmen verbundenen Kapitalisierung - bei der Kapitalisierung von Reserven handelte es sich vielfach nur um einen buchtechnischen Vorgang - war der Fortschritt der Comunidad Industrial in der Beteiligung am Kapital der Unternehmen gering. Ende 1970 wiesen die Unternehmen ein Grundkapital von 9,2 Milliarden S/. auf, das sich bis Ende 1971 um 56,8\% auf 14,4 Milliarden S/. erhöhte. Bis Jahresende 1972 nahm das Grundkapital nochmals um 16,6\% zu und betrug somit 16,8 Milliarden S/. Die 16,8 Milliarden S/. bezahltes Grundkapital setzten sich wie folgt zusammen: Nationales Kapital 37\%, ausländisches Kapital $60 \%$, Comunidad Industrial 3\%. Der Kapitalanteil der Comunidad Industrial belief sich Ende 1970 auf 22 Millionen S/. und steigerte sich in den genannten Zeiträumen auf 159 bzw. 515 Millionen S/. Er erhöhte sich damit von 0,34\% auf 3,0\%33. Die Gewinnbeteiligung verlief im Zeitraum 1971/ 1972 folgendermaßen: Die 132 Unternehmen machten 1971 Gewinne in Höhe von 4,0 Milliarden S/., 1972 wurden 4,9 Milliarden, also $21 \%$ mehr erzielt. Davon entfielen 1971 rund 728 Millionen S/. (18,1\%), 1972 etwa 913 Millionen S/. (18,8\%) auf die Arbeitnehmer, und zwar in Form von ausgezahlten $(10 \%)$ und kapitalisierten $(15 \%)$ Gewinnanteilen sowie Dividenden und entsprechenden Anteilen an der Reinvestition. Die „socios capitalistas“ partizipierten 1971 mit 63,7 \% (2,6 Milliarden S/.) am Gewinn, 1972 mit 62\% (3,0 Milliarden S/.). Der Rest entfiel in Form von direkten Gewinnsteuern und der $2 \%$ igen Technologieabgabe auf den Staat. Der Gewinnanteil pro Arbeiter ${ }^{34}$ erhöhte sich von 1971 (19045 S/.) auf 1972 (20 873 S/.) um 18,2\%. Die Redistributionseffekte dieser kombinierten Form

\footnotetext{
32 Vgl. dazu u. a. MIT, Principales discursos ... . Lima, Enero 1974, S. 64.

33 Uber die Kapitalbeteiligung sämtlicher anerkannter Comunidades Industriales liegen nur grobe Schätzungen vor. Sie wurde Anfang 1974 mit durchschnittlich 6,9\% am Grundkapital beziffert, erreichte jedoch in einigen Branchen zwischen 10 und $13 \%$ (Möbel, Gummi, Metallprodukte, Schuhe und Konfektion). Der Industrieminister gab Anfang Mai 1974 bekannt, daß die Eigentumsbeteiligung in einigen Firmen bei $25 \%$ liege; im Durchschnitt 8 bis $9 \%$ erreiche (Oiga, 24. 5. 1974). Mittels mathematischer Modelle wurde errechnet, daß die Comunidades Industriales - vor allem je nach Entwicklung des steuerpflichtigen Nettogewinns und in geringerem Maße beeinflußt von der Reinvestitionspolitik - nach etwa 10 bis 30 Jahren $50 \%$ Kapitalbeteiligung erreicht haben wird. (Vgl. z. B. Pedro de las Casas Grieve u. a. Análisis de la participación de la Comunidad Industrial en el capital social de la empresa, Lima 1970; David Llarena G. u. a., La Comunidad Industrial: Naturaleza económica de la empresa industrial en el Perú, Lima 1972.)

34 Kapitalisierung $(15 \%)$ und Gewinnausschüttung $(10 \%)$.
} 
von Kapitalbeteiligung sowie Gewinn- und Dividendenausschüttung sind dreimal so hoch wie die traditionelle Gewinnbeteiligung ${ }^{35}$. Die analysierten Unternehmen reinvestierten 1971 und 1972 durchschnittlich 32,1\% (1,2 Milliarden S/.) bzw. $36,7 \%$ (1,8 Milliarden S/.) der Gewinne. Daran partizipierte die Comunidad Industrial mit 36,6\% (472 Millionen S/.) und 30,5\% (545 Millionen S/.). Der Zuwachs der Reinvestition der Comunidad Industrial beläuft sich auf 15,5\% gegenüber einer Gesamtzunahme der Reinvestition um 38,3\%. Der geringe Zuwachs in der Reinvestitionsquote der Comunidad Industrial und die relative Abnahme in der Beteiligung sind Konsequenz der stark angestiegenen Reinvestitionsquote der "propietarios capitalistas“ (1971: $32 \% ; 1972$ : 41,1\%).

\section{Konflikte bei der Gründung und Durchsetzung der Comunidad Industrial - Unternehmerseite}

In den vergangenen Jahren kristallisierte sich eine Reihe von ständig wiederauftretenden Konflikten in bezug auf die Gründung und Durchsetzung der Comunidad Industrial heraus, die je nach Betriebsgröße, Betriebstyp, Management, Kapitaleigner etc. in Ausprägung und Intensität variieren. Die Hauptursachen für die Konflikte liegen in Gesetzeslücken und -kontradiktionen, in mangelnder Kontrolle und fehlenden rechtlichen $Z$ wangsmechanismen. Die langfristig eventuell denkbare Milderung der Konflikte zwischen Kapital und Arbeit ${ }^{36}$ durch die Comunidad Industrial passiert kurzfristig ein Stadium verschärfter Positionskämpfe zwischen ursprünglichen und neuen Eigentümern. In der Mehrzahl der Konflikte versuchen die Unternehmer die Bestimmungen des D. L. 18384 zu umgehen bzw. seine Auswirkungen zu Gunsten der Arbeitnehmer zu boykottieren.

\section{a) Mechanismen der Boykottierung der Comunidad Industriai}

Das Industrieunternehmen gründet eine Dienstleistungsfirma oder nimmt die Dienste einer bestehenden Firma in Anspruch. In Unternehmen des Dienstleistungsgewerbes existiert weder eine Gewinnbeteiligung (10\%) dieser Form noch Kapitalbeteiligung $(15 \%)$ bzw. keine Institution entsprechend der Comunidad Industrial. Mögliche negative Konsequenzen sind:

- Ein Teil der Arbeitnehmer wird z. B. von der Dienstleistungsfirma übernommen und partizipiert dann weder an Gewinnen noch an progressiver Kapitalbeteiligung in der Industriefirma ${ }^{37}$.

- Die Aufwendungen für die Dienstleistungsfirma erhöhen die Kosten und vermindern den Gewinn des Industrieunternehmens. Der Nettogewinn als Bemessungsgrundlage für die Comunidad Industrial wird geringer.

- Durch die „Umdisposition“ der Arbeitnehmer wird die gewerkschaftliche Einheit der Betriebsangehörigen unterhöhlt bzw. die gewerkschaftliche Organisierung unterbunden, wenn die Arbeitnehmerzahl unter 20 sinkt.

\footnotetext{
35 Es wird von der Hypothese ausgegangen, daß traditionelle Gewinnbeteiligung $10 \%$ am steuerpflichtigen Nettogewinn sind, wie sie in einigen Unternehmen praktiziert wurde.

36 Eine Harmonisierung von Kapital und Arbeit scheint nicht möglich, solange „50 zu 50\%-Gruppen bestehen bleiben, und keine Integration herbeigeführt wird.

37 Art. 52 von D. L. 18384 verfügt allerdings, daß die. Beschäftigten von Dienstleistungsunternehmen, die in „forma estable ${ }^{\alpha}$ in einem Industrieunternehmen arbeiten, Mitglieder der Comunidad Industrial sind.
} 
Ảhnlich sind die Auswirkungen bei einer Handelsfirma, wenn sie Eigentum derselben Aktionäre ist. In diesem Fall kann die Industriefirma ihre Produkte praktisch zum Kostenpreis an die Handelsfirma abgeben. Sie macht dann nur geringen oder keinen Gewinn, an dem die Comunidad Industrial partizipieren kann. Ein vielfach von mittleren und kleinen Unternehmen praktizierter Mechanismus ist die Teilung der Industriefirma in Betriebsgrößen, in denen entweder die gewerkschaftliche Organisierung, die starken Einfluß auf die Durchsetzung der Comunidad Industrial hat, zerschlagen oder die gesetzliche Grenze für die Comunidad Industrial unterlaufen wird. Betrügerischer Bankrott ist eine andere Form, um das Industrieunternehmen und damit die Comunidad Industrial zu liquidieren.

Häufiger als Mechanismen, die direkt gegen die Einführung der Comunidad Industrial zielen, sind z. B. verschiedene Praktiken, um den steuerpflichtigen Nettogewinn zu verringern. Das geschieht durch:

- Ankauf von Maschinen und Ausrüstungen, der von der Unternehmensentwicklung her nicht begründet ist;

- Verkauf von Produkten zu einem höheren Preis als in den Rechnungen ausgewiesen wird;

- Verkauf ohne Rechnung;

- Überhöhung von Kostengruppen mittels Einsetzen von privaten Ausgaben;

- Führen von „trabajadores fantasmas“ und damit fingierten Lohn- und Gehaltskosten;

- Überhöhung von Gratifikationen und sonstigen Extrazahlungen vor allem für leitende Angestellte sowie sehr hohe Gehälter für die Kapitaleigner, die in der Firma arbeiten. Das Gesetz sieht keine Maximalbeträge vor.

Ferner versucht man von seiten der Unternehmer den Kapitalbeteiligungsprozeß der Comunidad Industrial zu verlangsamen, in dem verstärkt reinvestiert wird; Kapitalerhöhungen durch rein buchtechnische Vorgänge erfolgen; Aktienübertragungen verzögert werden, wodurch die Comunidad Industrial nicht nur der anfallenden Zinsen und Dividenden verlustig geht, sondern auch die Partizipation an der Unternehmensführung hinausgeschoben wird. Bei der Neuausgabe von Aktien kommt den Unternehmen ein sehr kompliziertes und langwieriges Verfahren gemäß der Ley de Sociedades Mercantiles entgegen. Die Bewertung der Aktien erfolgt zum Nominalwert ${ }^{38}$, so daß ein Sinken des Realwertes unter die genannte Grenze die Comunidad Industrial benachteiligt, da sie für den 15\%igen Anteil am Nettogewinn entsprechend weniger Aktien bekommt. Häufigster „Trick“ ist die Präsentierung von frisierten Bilanzen, in denen seit Bestehen der Comunidad Industrial „Verlust“ ausgewiesen wird.

Ergebnis der gesamten Maßnahmen ist, daß drei Jahre nach Erlaß des Gesetzes noch immer etwa $10 \%$ der Unternehmen keine Comunidad Industrial haben; daß sich die Beschwerden und Denunzierungen durch Vertreter der Comunidad Industrial beim MIT über den Boykott der gesetzlichen Bestimmungen häufen; daß die Kapitalbeteiligung nur langsam fortschreitet und der Arbeitskampf sich in den Firmen verschärft hat.

38 Die Bewertung der Aktien erfolgt zum Nominalwert, wenn sie aus Reinvestitionen stammen; zum Realwert, wenn es sich um den Kauf von Anteilen der Kapitaleigner handelt. 


\section{b) Probleme bei der Beteiligung der Comunidad Industrial an der Unternehmensführung}

Weitaus größere Schwierigkeiten als die ökonomische Beteiligung der Arbeitnehmer wirft jedoch ihre Partizipation an der Unternehmensführung auf. Die Ursache ist bei vielen, vor allem kleineren und mittleren Unternehmen, in einer sehr engen Bindung zwischen Eigentum und Unternehmensleitung zu sehen sowie in dem sich nur sehr langsam vollziehenden "Umdenkungs- und Umerziehungsprozeß“, was Rechte und Pflichten gegenüber dem Unternehmen betrifft, die jetzt von Kapital und Arbeit gemeinsam ausgeübt werden sollen. Häufigste "Tricks" zur Umgehung der Bestimmungen sind:

- Die Vertreter der Comunidad Industrial werden gar nicht oder zu spät zu den Sitzungen des Vorstandes eingeladen. Das wurde von fast $50 \%$ der 332 befragten Vertreter der Comunidad Industrial bestätigt.

- Mit den Vertretern der Comunidad Industrial werden „fingierte“ Vorstandssitzungen abgehalten.

- Der Vertreter der Comunidad Industrial wird nicht am Kommunikationsfluß beteiligt; er erhält nicht rechtzeitig oder gar nicht die notwendigen Unterlagen. Die Umfrage ergab folgendes Bild zu dem Punkt:

$\begin{array}{lcc}\text { Aushändigung von } & \mathrm{Ja} & \text { Nein } \\ \text { Statuten } & 31 \% & 69 \% \\ \text { Kopien der Vorstandssitzungsprotokolle } & 28 \% & 72 \% \\ \text { Tagesordnung für die Vorstandssitzungen } & 22 \% & 78 \% \\ \text { Kopien der Bilanzen } & 64 \% & 36 \%\end{array}$

- $81 \%$ der Befragten bestätigten, daß den Vertretern der Comunidad Industrial die vorgeschriebene Freistellung von der Arbeitszeit gewährt sowie entsprechender Versammlungsraum zur Verfügung gestellt werden. Diese „Zugeständnisse“ tangieren die Unternehmensführung nicht und sind somit leicht durchsetzbar;

- Da die Partizipation an der Unternehmensleitung gemäß Kapitalbeteiligung zunimmt, wird versucht, die Comunidad Industrial anteilsmäßig so lange wie möglich in der Minorität zu halten, für deren Schutz keine gesetzliche Garantie besteht.

\section{c) Stellungnahme der Sociedad de Industrias zur Comunidad Industrial}

Die Sociedad de Industrias, Sprecher der Groß- und Mittelindustrie in Peru und früher eine der mächtigsten Verbände, die trotz Aberkennung ihres offiziellen Status durch die Regierung und Verbannung ihres Präsidenten aus Peru noch immer einigen Einfluß hat, nimmt in offiziellen Verlautbarungen zurückhaltend und vorsichtig zur Comunidad Industrial Stellung.

Die Sociedad Nacional de Industrias betonte im Januar $1972^{39}$, daß sie nicht die Abschaffung der Comunidad Industrial fordere sondern Veränderungen, die dieses Instrument den Notwendigkeiten der peruanischen Realität und Entwicklung anpaßt. In bezug auf Veränderungen regt sie an, u. a. folgende Probleme zu überdenken:

39 Im Januar 1972 war die Sociedad de Industrias noch als offizielle Vertretung der Industrie Perus von der Regierung anerkannt und führte das Wort "Nacional ${ }^{\alpha}$ in ihrem Namen. 
- Kollektivierung versus Entfaltung individueller Unternehmerinitiative;

- Mitbestimmung in der Unternehmensführung versus negativer oder positiver Beeinflussung der Entwicklung des Unternehmens;

- Verbesserte Einkommens- und Lebensbedingungen für die Arbeitnehmer versus Zeitraum für das Erreichen dieses Ziels;

- Rückgang des Quotienten Gewinn/Kapital für den Investor versus Beteiligung der Arbeitnehmer und des Staates am Unternehmensergebnis.

Die zunehmende „Abkühlung“ der Beziehungen zwischen der Privatindustrie bzw. der Sociedad de Industrias und der Regierung im Jahre 1973 sowie das dem Verband drohende Verbot bewirkten insofern eine Anpassung an den Kurs der Regierung, daß die Sociedad de Industrias 1974 erklärte, man wolle ein Beteiligungsund Mitbestimmungssystem unterstützen, sofern die Stabilität der Unternehmen nicht leide und die Rentabilität für den Kapitalgeber nicht weiter zurückgehe. Sollten diese Bedingungen nicht erfüllt sein, wäre eine weitere Abnahme der Investitionsneigung der Privatindustrie und eine bremsende Wirkung auf die Industrialisierung unvermeidlich. Hauptargument des privaten Sektors gegen den D. L. 18384 ist, daß das Gesetz - so wie es zur Zeit besteht - ein Instrument zur Unternehmensführung mit nur transitorischem Charakter ist, und zwar bis diese Unternehmen in Selbstverwaltung (autogestión) der Arbeitnehmer übergehen. Diese Annahme entbehrt nicht einer gewissen Realität, wenn man z. B. die Verabschiedung des Gesetzes über die Propiedad Social, die Verlautbarungen der Regierung zur Politik gegenüber diesem neuen Sektor, die Forderungen der Vertreter der Comunidades Sociales in Rechnung stellt. Die Privarindustrie sieht nicht nur den ihr offiziell zugestandenen Betätigungsrahmen de facto immer enger werden, sondern fürchtet auch, daß sie aufgrund der prioritären Förderungen der anderen Eigentumsformen durch den Staat an Konkurrenzfähigkeit einbüßt bzw. in Bereiche abgedrängt wird, die ihr nicht die „notwendige“ Rentabilität garantieren.

Die Sociedad de Industrias stellt drei grundlegende Mängel der Comunidad Industrial heraus:

- In D. L. 18350 wird das Problem der Unternehmens- und Eigentumsreform sektoral und als Apendix zu Industrieförderungsbestimmungen gelöst, ohne den Sektor insgesamt sowie andere Sektoren der Wirtschaft integral und organisch einzubeziehen, um als Resultat der Reformmaßnahmen eine gerechtere Einkommensverteilung und eine Stärkung der Unternehmen zu erzielen. Beide Ziele werden aufgrund dieses partiellen Ansatzes nicht erreicht. Eine privilegierte Arbeiterschaft in der Industrie entsteht, die Wachstum und Rentabilität der Unternehmen beeinträchtigt.

- Die Grenzen für die Beteiligung der Comunidad Industrial sind zu weit gezogen. Dem Gesetz liegen keine realistischen Studien und Annahmen über die Rentabilität des Sektors zugrunde. Verdient ein Unternehmen 30\% über das Grundkapital, so bleiben nach Abzügen für Comunidad Industrial und den Instituto de Investigación Tecnológica Industrial y Normas Técnicas (insgesamt $27 \%$ ) $73 \%$, auf die Gewinn- und Dividendensteuer anfallen. Der endgültige Betrag, der zur Verfügung steht, beträgt etwa $9 \%$, gerechnet auf das Grundkapital. Das bedeutet, wenn ein Anteil verteilt werden soll, müssen drei verdient werden. Bezieht man die notwendigen Reinvestitionen ein, nimmt der verteilungsfähige Gewinn weiter ab. 
- Die Festsetzung der Beteiligung am Kapital und an der Unternehmensführung auf ein „50 zu 50 Verhältnis“ ist nicht funktionsfähig. Nach dem heute gültigen Aktienrecht könnte, wenn die Comunidades Industriales in den Unternehmen die 50\%-Grenze erreicht haben, der Fall eintreten, daß ein um das andere Jahr in den Betrieben entweder die „socios capitalistas“ oder die „socios comuneros" den Präsidenten des Vorstandes stellen. Diese Regelung kann nach Ansicht der Sociedad de Industrias zu chaotischen Zuständen nicht nur in der Entwicklung der Unternehmen sondern des gesamten Sektors führen. Ist die 50\%-Grenze erreicht, besteht zudem ein geringer Anreiz für die private Unternehmertätigkeit. Bei $30 \%$ Gewinn entfallen $4,5 \%$ auf die ursprünglichen Aktionäre, $12 \%$ auf die Comunidad Industrial (sie erhält weiter die $10 \%$ bzw. $15 \%$ vom Nettogewinn), 12,9\% gehen an den Staat und 0,6\% an das Technologieinstitut.

\section{Schwierigkeiten bei der Durchsetzung der Comunidad Industrial - Arbeitnehmerseite}

Die Ley de Comunidad Industrial gibt den Arbeitnehmern in Peru erstmals das Recht der Kapitalbeteiligung und der darauf basierenden Partizipation an der Unternehmensführung. Auf diese neuen Rechte ist die Masse der Beschäftigten in der Industrie sowohl von der Vor- als auch von der Ausbildung her nicht oder sehr ungenügend vorbereitet. Die „comuneros“ wie die von ihnen gewählten Vertreter sind mit ihren neuen Rechten „überfordert“. Es kommt zum „Ausleben“ von angestauten und sich fortwährend neu bildendem Mißtrauen, zu einem „Überziehen“ der den „comuneros“ zustehenden Rechte, die zum Teil in Befehlsverweigerungen und offenen Aggressionen enden. Die Comunidad Industrial umschließt als Mitglieder mit entsprechenden Rechten und Pflichten die verschiedenen Beschäftigungsgruppen, die im Unternehmen normalerweise in ein hierarchisches, vertikal organisiertes Macht- und Entscheidungssystem eingebunden sind, aus dem Zersplitterung und Spannungen zwischen den Betriebsangehörigen resultieren. Diese setzen sich naturgemäß in der Wahrnehmung der Rechte und Pflichten der Comunidad Industrial fort und behindern deren Durchsetzung und Funktionieren erheblich.

Die Motivation der Arbeitnehmer, Pflichten zu erfüllen, die sich aus der Comunidad Industrial ergeben, ist noch gering ausgebildet. Nur die Pflichtzahl an Versammlungen pro Jahr wurde nach Auskünften der 332 Vertreter der Comunidad Industrial im Durchschnitt in allen Branchen eingehalten, obwohl anzunehmen ist, daß die auftretenden Mängel des Systems häufigere Versammlungen notwendig machen. Die Teilnehmerzahl bewegte sich zwischen $53 \%$ (Nahrungsmittel) und $80 \%$ (diverse Industrien) der "comuneros“. Sie war in den kleineren Betrieben aufgrund des engen Kontaktes der Beschäftigten, der Kontrolle untereinander höher als in den großen Unternehmen. Die eigene Beurteilung der Comunidad Industrial über ihre Partizipationsausübung läßt erkennen, daß dieses Recht bis jetzt kaum oder nur sehr unvollkommen wahrgenommen wird. $60 \%$ der befragten Präsidenten antwortete, daß die Comunidad Industrial wenig oder gar nicht in die Unternehmensführung eingreift. Der gleiche Prozentsatz stellte fest, daß die Beteiligung der "directores comuneros“ (Mitglieder der Comunidad Indu- 
strial im Vorstand des Unternehmens) stark zunehmen muß. Nur 59\% waren der Ansicht, daß die Comunidad Industrial die Unternehmensführung erleichtert. Allerdings unterstrichen $75 \%$, daß durch die Comunidad Industrial eine Produktionserhöhung eintreten wird.

Eine skeptische und von großer Unsicherheit gekennzeichnete Einstellung gegenüber der Comunidad Industrial ist bis in die höchsten Führungsposten zu beobachten. $73 \%$ der Präsidenten der Comunidad Industrial gaben an, daß ihnen diese Einrichtung geringe oder keine Vorteile brächte. $35 \%$ nimmt an, daß ihre Einkommenssituation gleich bleibt, sich verschlechtert bzw. hatten zu dieser Frage keine eindeutige Meinung. $40 \%$ glaubt nicht, daß mit der Comunidad Industrial die Rechte und Interessen besser als früher verteidigt werden. Für diese wenig überzeugte und abwartende Haltung der Präsidenten der Comunidades Industriales sind u. a. zwei Faktoren in Rechnung zu stellen: 1. Die Antworten stammen aus dem Jahre 1972, d. h. die Comunidad Industrial dürfte sich in der Zwischenzeit etwas mehr gefestigt haben. 2. Die Befragten gehören nach Alter $(36 \%$ zwischen 20 und 30 Jahren), Beschäftigungsverhältnis (48\% Arbeiter), Einkommen (69\% unter $6000 \mathrm{~S} /$. pro Monat) und Ausbildung (36\% nicht beendete oder beendete Grundschule) nicht $\mathrm{zu}$ den favorisierten Gruppen ${ }^{40}$ der Industriebeschäftigten. Ihre Skepsis und Unsicherheit ist u. a. Ergebnis einer jahrelangen Marginalisierung und Frustration über nicht durchgeführte Reformmaßnahmen.

Die Durchsetzung der Comunidad Industrial wurde in vielen Unternehmungen durch mit den Gewerkschaften auftretende Spannungen behindert. Die Gewerkschaften als traditionelle Vertretung der Rechte und Forderungen des Faktors Arbeit fürchten eine Einbuße von Macht und Einfluß durch die Herausbildung einer neuen Führungsgruppe innerhalb der Arbeiterschaft und die eventuelle Annäherung zwischen Arbeit und Kapital mittels der Comunidad Industrial. Von den befragten Präsidenten der Comunidad Industrial unterstrichen $75 \%$, daß sie die Gewerkschaft für wichtig oder sehr wichtig halten. Allerdings mißt die Hälfte der Befragten der Comunidad Industrial mehr Gewicht bei, da sie stärker von den Arbeitnehmern unterstützt wird.

Die Strategie der Gewerkschaften war in den vergangenen Monaten daraufgerichtet, die Kooperation mit den Comunidades Industriales zu stärken bzw. durch "Gewerkschaftsanhänger" die Organe der Comunidad Industrial zu dominieren und zu majorisieren. Geht man davon aus, daß die ,50 zu 50 Beteiligung “ in den Unternehmen bestehen bleibt, und eine Identifizierung zwischen Kapital und Arbeit nicht oder nur in Einzelaspekten stattfindet, so werden die Gewerkschaften als Instrument zur Durchsetzung von Lohnforderungen und Sozialleistungen und die Comunidad Industrial als Organ der Partizipation in den Unternehmen koexistieren. Diese Annahme ist vor allem gerechtfertigt, wenn es entweder zur Durchsetzung der Gremien der Comunidad Industrial mit Gewerkschaftsmitgliedern kommt oder die beiden Institutionen sich auf eine gegenseitige Abstützung einigen ${ }^{41}$. Beide Entwicklungen bahnen sich an.

\footnotetext{
40 Denkbar ist z. B. daß Angestellte oder "profesionales (Akademiker, Techniker) aufgrund ihrer Erziehung und Ausbildung die Führungsrolle übernehmen. Jedoch ist fraglich, ob ihre Motivation und Interessenlagen mit denen der Arbeiter, die die Masse der Beschäftigten stellen, harmonieren.

41 Als Beispiele seien die Unternehmen Backus y Johnston S. A. und Astilleros PICSA genannt, in denen einerseits die Comunidad Industrial die Lohnforderungen der Gewerkschaften unterstützte, andererseits die Gewerkschaft mit der Comunidad Industrial deren Recht durchzusetzen suchte, daß die Beschäftigten des Dienstleistungsbereiches zur Comunidad Industrial gehören.
} 
Wie schon an anderer Stelle erwähnt, hat der Arbeitskampf im Industriesektor seit Bestehen der Comunidad Industrial nicht abgenommen sondern sich verschärft. 1973 fanden 409 Streiks statt. Das sind 156 (62\%) mehr als 1972. Von den Streiks wurden etwa 80400 Arbeitnehmer erfaßt; 4,3 Millionen Arbeitsstunden pro Person gingen verloren. Die Ursachen dürften in einer verstärkten Kampfhaltung der Gewerkschaften ${ }^{42}$ aufgrund der gefürchteten Harmonisierung zwischen Arbeit und Kapital sowie in der sich verhärtenden Konfliktsituation zwischen Arbeitnehmern und Arbeitgebern durch die Boykottierung der Comunidad Industrial zu suchen sein. Nach Aussagen von Experten wurden etwa $60 \%$ der Streiks durch keinen echten, nicht durch Verhandlungen beilegbaren Arbeitskonflikt hervorgerufen, sondern entstanden durch die verhärteten Positionen der beiden Seiten vor allem bei Lohn- und Personalfragen.

\section{Ausbildung und Schulung der „comuneros“ als Voraussetzung der Kooperation im Unternehmen}

Ein wesentlicher Engpaßfaktor für die Durchsetzung der Comunidad Laboral ist die mangelhafte Ausbildung und Schulung der "comuneros“, vor allem in sämtlichen Fragen der Unternehmensleitung. Mit D. S. 021-72-IC/DS wurden die zuständigen Fachministerien mit der Ausbildung der "comuneros“ betraut, die sie in Koordination untereinander und/oder mit SINAMOS ${ }^{43}$ durchführen sollen.

D. L. 19339 beauftragt die Oficina de Comunidades Laborales (OCLA) des Ministerio de Industria y Turismo mit der Koordinierung, Beratung und Ausbildung der Comunidades Industriales. Ziel ist, die Comunidades Industriales zu einem effizienten Instrument des strukturellen Reformprozesses in Peru zu entwickeln ${ }^{44}$.

Auf dem Gebiet der Ausbildung ${ }^{45}$ fanden 1971 für 407 Comunidades Industriales mit etwa 10000 Mitgliedern Kurzkurse in Lima-Callao und Arequipa statt, um die grundlegenden Bestimmungen der Gesetzesdekrete 18350 und 18384 zu verbreiten. 1972 wurde diese allgemeine Informationstätigkeit auch auf andere Gruppen wie Journalisten, Studenten ausgedehnt. Erste Ausbildungskurse, in denen noch der Motivations- und Aufklärungscharakter überwog, fanden statt. Im gleichen Jahr schuf ein Intensivkurs für "promotores ${ }^{46 "}$ und "supervisores" 4 " des MIT eine erste Grundlage für die systematischere Ausbildungsarbeit. Etwa 50000 Personen nahmen an den Veranstaltungen 1972 teil. Das Ausbildungsprogramm 1973/74 wurde im Rahmen eines Zweijahresplanes durchgeführt, der eine systematische und eine begleitende Schulung (educación asistemática) vorsieht. Bei der systematischen Ausbildung werden die "comuneros" in sozio-ökonomischen, technischkaufmännischen, politischen und rechtlichen Fragen, die direkt oder indirekt

\footnotetext{
$42 \mathrm{Da}$ der zunehmende Arbeitskampf für die Stellung der Militärs gefährlich werden kann, gründete die peruanische Regierung zur Kontrolle der Gewerkschaften und als Gegengewicht zur von der KP beherrschten Confederación General de Trabajadores del Perú die CTRP (Central de Trabajadores de la Revolución Peruana)

43 SINAMOS (vgl. FN 6) als Organ zur politischen Mobilisierung und Organisierung der Basisorganisationen nimmt mittels der "Unidad de Organizaciones Laborales". ebenfalls Ausbildung und Schulung der "comuneros" vor, bisher. jedoch weitgehend auf politisch-ideologischem Gebiet und mit besonderer Intensität außerhalb von Lima-Callao.

44 Die Koordinierung zwischen MIT und SINAMOS ist ungenügend, auch aufgrund der mangelhaften

45 Die OCLA verfügt über folgende Abteilungen: División de Registro (9 Personen), División de

Supervisión (28 Personen), División de Capacitación (37 Personen).

46 Promover: fördern.

47 Supervisar: überwachen.
} 
die Comunidad Industrial berühren, geschult ${ }^{48}$. Bis September 1973 durchliefen rund 5300 „comuneros“ aus 800 Comunidades Industriales diese Kurse. Die begleitende Ausbildung mittels Vorträgen, Gesprächen, Kurzkursen erreichte ca. 550 Comunidades Industriales mit 25000 Mitgliedern.

Die Evaluierung der Ausbildungsarbeit der OCLA bis Ende 1973 ergibt:

- Gute Fortschritte in der Arbeit mit Motivations- und Informationscharakter.

- Für die systematische Ausbildung müssen differenzierte, mit angepaßten Lehrund Lerninhalten sowie Lehrmitteln arbeitende Programme entwickelt werden.

- Personal- und Organisationskapazität sind unzureichend, um größere Fortschritte in der systematischen Ausbildung der "comuneros" zu machen ${ }^{49}$.

- Die Arbeit der OCLA in den Zentren außerhalb Limas muß verstärkt werden.

- Die Unternehmer, vor allem der kleineren Betriebe, müssen in das Ausbildungsprogramm integriert werden.

- Die Koordinierung und Kooperation mit SINAMOS muß entscheidend verbessert werden.

- Planziel der OCLA ist, pro Jahr etwa 20000 Unternehmer und „comuneros“ von der begleitenden Schulung zu erfassen sowie 35000 comuneros systematisch auszubilden.

Die Ausbildungsprogramme werden durch einen Beratungsservice ergänzt, der in Quantität und Qualität der Verbesserung bedarf. Im Jahre 1972 wurden in der OCLA etwa 10300 Fälle und „Klagen“ präsentiert, von denen rund 3700 in den regelmäßigen Sprechstunden vorgetragen wurden. Aus einer Klassifizierung von 1800 Fällen geht hervor, daß rund 730 sich auf Gewinn- und Vermögensverteilung, sowie Bilanz- und Buchprüfungsvorgänge bezogen. Nur in 9 Fällen wurde in der OCLA um Beratung und Ausbildung nachgesucht. Wiederum ein Indiz, daß die Comunidad Industrial noch in den Anfängen steckt. 1973 (Januar bis September) wurden 3099 Fälle in den Sprechstunden präsentiert, davon bezogen sich 29,7\% auf Verwaltung, Kontrolle und Leitung der Comunidad Industrial; $12 \%$ auf Bilanz und Buchhaltung, 11, 2\% auf Gewinnverteilung.

\section{Ergebnisse des I. Nationalen Kongresses der Comunidades Industriales}

Vom 23. 2. bis 2. 3. 1973 fand der I. Kongreß der Comunidades Industriales, organisiert vom MIT und SINAMOS, auf nationaler Ebene statt. Unter fast paramilitärischer Abschirmung übten die Vertreter der Comunidades Industriales harte „Manöverkritik“. Sie brachten Hunderte von Anregungen und Vorschläge ein, die in einer gemeinsamen Resolution mit etwa 150 Beschlüssen zusammengefaßt wurden. Direkt zur Institution der Comunidad Industrial forderten die Vertreter u. a.:

- Detaillierte Studie über die Einführung der Comunidad de Compensación (Ausgleichsfonds) im Industriesektor ${ }^{50}$.

\footnotetext{
48 Der Instituto Peruano de Administración de Empresas (IPAE) wurde von der OCLA beauftragt, systematische Ausbildungskurse für "comuneros abzuhalten. Ein Teil der Finanzierung wird von der Friedrich-Naumann-Stiftung getragen.

49 Vgl. Kritik auf dem I. Kongreß der Comunidades Industriales.

50 Die Comunidades Industriales der größeren Betriebe wehren sich gegen die Einführung eines Ausgleichsfonds, der ihre Kapital- und Gewinnbeteiligung schmälert. Eine ähnliche Haltung ist bei allen Comunidades Industriales in bezug auf Einstellung von zusätzlichem Personal zu beobachten. Jeder neue Betriebsangehörige reduziert den Gewinn- und Kapitalanteil.
} 
- Aufbau eines Instituts zur Ausbildung der „comuneros“, um das Monopol der OCLA zu brechen und diese Arbeit verstärkt und zügiger voranzutreiben.

- Gründung der Comunidad Laboral in allen Wirtschaftssektoren und Verstärkung der Mitbestimmung sowie Kontrolle der Comunidad Industrial über die Unternehmen.

- Grundlegende Reorganisierung der Oficina de Comunidades Laborales des MIT und verstärkte Dezentralisierung der für die Comunidad Industrial zuständigen Institutionen.

- Größere Unterstützung der Comunidades Industriales durch SINAMOS.

- Einräumung von speziellen Kreditlinien durch COFIDE und den Banco Industrial del Perú, um z. B. durch Aufkauf von Aktienanteilen den Kapitalanteil der Comunidad Industrial am Unternehmen schneller zu vergrößern ${ }^{51}$.

- Gründung einer nationalen Organisation der Comunidades Industriales ${ }^{52}$.

- Verbot der Sociedad de Industrias.

- Anderung aller Gesetze (wie z. B. Aktien- und Konkursrecht), die der effektiven Durchsetzung der Comunidad Industrial entgegenstehen.

- Einrichtung eines speziellen Gerichts zur Beilegung von Streitigkeiten zwischen Unternehmen und Comunidad Industrial ${ }^{522}$.

- Modifizierung der bestehenden Ley de Comunidad Industrial (D. L. 18 384) unter Berücksichtigung der Erfahrungen der letzten zwei Jahre und der Beschlüsse des Kongresses.

Schon aus den genannten Beschlüssen ${ }^{53}$ wird sichtbar, daß die "comuneros" in gemeinsamer Aktion Druck ausüben, um die Comunidad Industrial zu einem Instrument der Unternehmensbeherrschung und -kontrolle auszubauen, um in $\mathrm{Zu}-$ kunft zu einer Selbstbestimmung (autogestión) in der Industrie zu kommen. Denn „die Comunidad Industrial . . . ist Teil einer bestimmten historischen Etappe und hat Übergangscharakter im Hinblick auf eine neue Gesellschaft, in der die Produktionsmittel soziales Eigentum ${ }^{54}$ sind. Der Fortschritt und die Radikalisierung der revolutionären Reformen bedingen, daß die Comunidad Industrial in ihrer augenblicklichen Form sich überlebt hat und die Einführung einer neuen Eigentumsform bedingt, die die Aspirationen, um die die Arbeiterklasse, allgemein das Volk kämpft, voll erfüllt ${ }^{55}$.

51 Ende Mai 1974 gewährte COFIDE der Comunidad von COPSA einen Kredit über 90 Millionen S/., mit dem die Comunidad ihren Anteil am Kapital von $12 \%$ auf $33,5 \%$ erhöhen kann. Sie erwirbt die aus der Kapitalerhöhung (Erweiterungsinvestition) ihr zustehenden Aktien. Das Unternehmen, eines der größten auf dem Ol- und Fettsektor, beschäftigt mehr als 600 Arbeitnehmer.

52 Noch auf dem Kongreß wurde die Confederación Nacional de Comunidades Industriales (CONACI) ins Leben gerufen, die heute 25 Föderationen in Lima und 8 in der Provinz umfaßt. Von dieser Organisation sind bis jetzt keine größeren Impulse ausgegangen. Mitte Mai 1974 wurde CONACI in "totaler Reorganisation befindlich ${ }^{\alpha}$ erklärt. Diese Restrukturierung, die z. T. interne Machtkämpfe "überdecken soll, feierte der "Expreso ${ }^{\alpha}$ als ein Beispiel demokratischer Beteiligung und Partizipation.

52a Bis heute existiert kein spezielles Gericht. Die OCLA hat Ưberwachungs- und Beratungsfunktionen, kann aber keine rechtswirksamen Entscheidungen treffen. Der Communidad Industrial steht nur der ordentliche Rechtsweg offen.

53 Eine detaillierte Wiedergabe sowie eine Analyse der Beschlüsse ist an dieser Stelle nicht möglich.

54 Das Gesetz über Propiedad Social wurde am 2. 5. 1974 verkündet.

55 Primer Congreso Nacional de Comunidades Industriales, 23. 2. bis 2. 3. 1973, Resolutionen, Lima 1973 (hektogr.), Punkt 7, S. 1. 
Zur Zeit wird in Peru auf höchster Ebene ein Entwurf zur Änderung des Gesetzes über die Comunidad Industrial diskutiert. Über die möglichen Änderungen sind folgende "Spekulationen“ im Umlauf:

- Einführung gesetzlicher Normen, um die "Praktiken“ der Boykottierung wie Teilung der Unternehmen, Gründung von Handels- und Dienstleistungsfirmen, künstliche Erhöhung der Kosten etc. zu unterbinden. Als Maßnahme ist z. B. denkbar, daß Fusion gesetzlich verbindlich wird, wenn die Industriefirma 50 Prozent des Kapitals von Dienstleistungs- oder Handelsfirmen in Besitz hat.

- Angleichung der Kapitalbildung der Comunidad Industrial bei den verschiedenen Eigentumstypen.

- Einführung eines Kompensationsfonds zwischen den Industrieunternehmen.

- Umwandlung der direkten Partizipation der Arbeitnehmer in den Kleinbetrieben (mögliche Abgrenzung: 6 bis 19 Beschäftigte und $300000 \mathrm{~S} /$. Anlagevermögen pro Beschäftigtem) in eine indirekte Form.

- Gesetzliche Zwangsmechanismen zur Verstärkung der Beteiligung der Arbeitnehmer an der Unternehmensführung.

Umstrittenster Punkt dürfte die Heraufsetzung der Betriebsgrößengrenze für die Einführung der Comunidad Industrial in ihrer jetzigen Form sein. In bezug auf die ausstehende Einbeziehung der kleinen Handwerksbetriebe (1-5 Beschäftigte) in das System der Mitbeteiligung und Mitbestimmung scheint sich keine Änderung anzubahnen, obgleich überall Kritik an der Privilegierung eines Teils der Industriearbeiterschaft geübt wird. Denn der Sektor ab 6 Beschäftigten macht nur 30 Prozent der Arbeitnehmer in der Industrie und 4 Prozent aller Erwerbstätigen in Peru aus.

\section{Schlußfolgerungen}

Die Analyse der grundlegenden gesetzlichen Bestimmungen der Comunidad Industrial, die Informationen über ihre Verwirklichung, über die auftretenden Konflikte und Spannungen zwischen traditionellen und neuen Gruppen, sektoralen und multisektoralen Institutionen lassen eine Vielzahl von wissenschaftlich relevanten Problemstellungen und Thesen für die verschiedensten Disziplinen zutage treten. Die im folgenden aufgeführten Thesen ${ }^{56}$ beziehen sich vorwiegend auf die Auswirkungen der Comunidad Industrial im Subsystem Wirtschaft:

- Die Comunidad Industrial bremst die Investitionstätigkeit in neuen Unternehmen.

- Investitionen in bestehenden Unternehmen zielen seit Einführung der Comunidad Industrial mehr als früher auf die Verstärkung der Kapitalintensität ab.

- Ausländische Investitionen im Rahmen des Grupo Andino werden durch die Comunidad Industrial in andere Mitgliedsländer "umgelenkt" oder unter „Durchlöcherung“ des Systems der Comunidad Industrial in Peru gebunden.

- Die Comunidad Industrial fördert den Gruppenegoismus der Arbeitnehmer und behindert die Ausweitung der Beschäftigung.

56 Die Thesensammlung erhebt keinen Anspruch auf quantitative oder qualitative Vollständigkeit. 
- Die Zahl der beschäftigungsintensiven Unternehmen der Mittel-, aber vor allem der Kleinindustrie, die ihre Kapazität relativ problemloser abbauen können, stagniert bzw. ist rückläufig. Neues Kapital legen diese Gruppen vielfach zwar in weniger rentablen, aber ihnen allein gehörenden Werten wie z. B. Rentenpapieren an.

- Auswirkung der aufgeführten Punkte ist, daß durch die Comunidad Industrial die Schaffung neuer Arbeitsplätze relativ abnimmt.

- Die Comunidad Industrial trägt nicht zur Redistribution des Einkommens innerhalb des gesamten Industriesektors bei. Sie vertieft den Einkommensunterschied zwischen "trabajadores artesanales ${ }^{57 "}$ und „trabajadores manufactureros $^{58 “}$ “. In der "industria manufacturera“ verteilt sie Eigentumsteile der Kapitaleigner auf die einkommenshöchste Schicht der Arbeitnehmer um.

- Organe und Funktionsweise der Comunidad Industrial sind nicht geeignet, eine Partizipation der Arbeitnehmer herbeizuführen, da sie auf dem Vertretungsprinzip ohne echte Integration der Arbeitnehmer in den Produktions- und Unternehmensprozeß beruhen.

- Die Comunidad Industrial führt nicht die Harmonisierung zwischen Arbeit und Kapital herbei. In dem „50 zu 50 System“ und ohne entsprechende Integrationsmechanismen werden Gewerkschaften und Lohnkämpfe fortbestehen.

- Durch die Comunidad Industrial verstärkt der Staat mittels sektoraler und multisektoraler Institutionen und Mechanismen Einfluß und Kontrolle über die Unternehmenspolitik des Privatsektors.

- Die Begründung der Propiedad Social (autogestión) und die ihr von der Regierung zugebilligte höchste Förderungspriorität stimulieren den Druck auf die Umwandlung der „empresa privada reformada“ und die Abnahme der privaten Unternehmertätigkeit.

- Der von der Regierung propagierte wirtschaftliche, wie auch der politische und kulturelle Pluralismus ist nur eine Übergangsform ${ }^{59}$. Der direkte und indirekte Abbau des Privatsektors sowie möglicherweise der „propiedad privada reformada" und der Genossenschaften ${ }^{60}$, das Vordringen der staatlichen Investitionen und der durch den Staat stark beeinflußten Propiedad Social signalisieren eine neue Form des Staatskapitalismus.

57 Betriebe bis 5 Beschäftigte.

58 Betriebe mit 6 Beschäftigten und mehr. D. L. 20681 vom 23. 7. 1974) - La Prensa wurde Zeitung der Comunidades Laborales - macht eine Gleichschaltung fast unvermeidbar.

60 Die Comunidades fordern - wie an anderer Stelle erwähnt - diese Umwandlung. Die Abschaffung der Genossenschaften und ihre Transformierung in Propiedad Social ist für den Industriesektor in der Strategie 1975 bis 1978 fixiert. 
Was the counterrevolution inevitable? Middle sectors and armed forces in Chile

\section{By Dieter Nohlen and Otto Boye}

The contribution of the two authors, who lived in Chile during the Allendegovernment, deals with the question, whether the Chilean way to socialism had a chance to be successful. It is argued that the democratic road to socialism was a valid concept for Chile. Its success depended mainly on the capacity of the socialist forces to attract middle sectors by responding to their claims and to their political attitudes. In elaborating the program of the Unidad-Popular-Government, the Chilean left and above all the Chilean Communist Party showed a clear conception of this necessity. The program favoured a policy leading to the neutralization of middle sectors and the armed forces.

The authors show how, during the Allende government, this conception was disregarded and finally completely abandoned by the Chilean left supporting the socialist government, so that Allende lost all possibilities to win general elections. Two factors led to this impass: The ideological and strategical contradictions within the left itself and the unbalanced economic policy, causing economic distortions. These factors favoured the unconditional opposition of groups within and outside Chile, as well as left wing socialists, proposing and preparing a violent solution of the political conflict. The armed forces intervened, when the Chilean way to socialism had lost any chance.

\section{Comunidad Industrial - an attempt to create a new structure and control of property in the peruvian industry}

\section{By Mechthild Minkner}

With the General Industries Law (D. L. 18350 of 1970) the peruvian revolutionary government initiated several reforms in the industrial sector, such as reservation of basic industries for public enterprises, promotion of industrial products according to a scale of priority, new regulations of foreign investment rules and participation of workers in industrial firms by the Comunidad Industrial.

The paper deals with the fundamental features and the development of the Comunidad Industrial as an instrument for creating the reformed private sector industrial firm. At first, on the base of the two main laws (D. L. 18350 and D. L. 18384) and the additional regulations the objectives, the operating structure and the organs of the Comunidad Industrial are analyzed. In concept, the Comunidad Industrial is a way for increasing both capital formation and production as well as for achieving conciliation between labor and capital. It establishes the participation of workers not only in profit and property but also in management. After that, guided by statistics, the range and the consequences of the concept are explained. As a matter of fact, the Comunidad Industrial up today has intensified the contradictions between labor and capital. In order to boycott the Comunidad Industrial the entrepreneurs are using different tools extending from deflating net income to fraudulent bankruptcy. Regarding the employees the realization of the Comunidad Industrial is mostly delayed by lack of educational background and instruc- 
tion. The motivation of the employees to contribute to a continuous strengthening and development of the firms is only small up to now, though they will be the owners of $50 \%$ of the capital of the firm in the future. As to the relations between owners and employees the struggle for higher wages and salaries, better social securities and stability of the labor market is still dominating. By an systematic training and teaching the competent public institutions are engaged with the improvement of the educational level and the comprehension of the employees for their new claims and duties.

The last two paragraphs are concerned with the requirements and the changes just discussed, which are necessary in order to improve the Comunidad Industrial. However, by some groups in Peru the reformed private sector industrial firm is regarded to be only a transitional stage leading to a self-managed property. Some conclusions are presented at the end of the paper. The main one states that the Comunidad Industrial in its present form is no feasible way neither for the full participation of all industrial employees nor for a redistribution of property in the industrial sector.

\section{Taxes and Tax administration in Costa Rica and Guatemala}

\section{By Hermann Avenarius and Jürgen H. WolfF}

Despite very complex tax systems, in practice, the income, sales, and import taxes plus excise tax in Costa Rica and petroleum tax in Guatemala account for the bulk of the total revenue: two thirds in Costa Rica, more than three quarters in Guatemala. Indirect taxation prevails; the tax load is on par with (Costa Rica) or below (Guatemala) the Latin American average. The tax systems are characterised by a lack of logics: so, a large number of duties just exists on paper (more so in Guatemala than in Costa Rica). There are many unjustified exemptions reflecting, e. g., the political strength of the landlords in Guatemala. Finally, despite progressive rates in Guatemala, a marked inelasticity of the tax revenue in relation to economic development must be observed. The administrative processes, in both countries, do not provide for any efficient control and cross-checking of the tax return sheets filled out by the taxpayers. Consequently, the State does not succeed in enforcing the tax laws in the measure to be desired. A special problem in Guatemala is the fact that, until now, no systematic codification of legal sources concerning taxes exists.

The organization of the tax administration in both countries is characterised by a marked degree of geographical and organisational centralisation. In Costa Rica as in Guatemala, the customs and internal revenue administrations are clearly demarcated within the Ministries of Finance. In Costa Rica, the size of the different departments charged with the collection of internal taxes are out of proportion with the revenue of the taxes. In Guatemala, the structure of the internal tax administration introduced in 1972 is complicated by combining functional and formal criteria.

Once more, the research underlying this paper has demonstrated that administrative problems of developing countries cannot be defined in technical terms alone: What is unsatisfactory from a purely technical point of view or when applying 